\title{
proguramación lineal aplicada al cálculo plástico elemental de estructuras lineales
}

\begin{abstract}
Expone este artículo un método para hacer los cálculos plásticos elementales de estructuras lineales con cargas puntuales por medio de programas Simplex, ya disponibles en cualquier ordenador científico, dando como resultado, sea un coeficiente de seguridad de una estructura ya dimensionada, sea una optimización de la misma. El cálculo es muy corto con un caso de cargas, y menos con varios casos de cargas. El método admite perfeccionamientos, que pueden hacer todavía más elemental su introducción en computador.
\end{abstract}

\section{Introduckión}

El cálculo plástico elemental es enseñado a los alumnos de Caminos (o equivalentes) en muchos países. La ${ }^{(1)}$ en la Biblioteca del Instituto Eduardo Torroja me sugirió que el cálculo plástico elemental se podía resolver por programación lineal. Escribí un comentario al artículo que será publicado en invierno del 70 en los Proceedings del A.S.C.E., habiendo sido aceptado (publicado abril 70).

Encontré después ${ }^{(2)}$ indicios de que ha sido tratado por su autor el problema. Se trata de M. Z. Cohn, Catedrático de la Universidad de Warteloo, Ontario, Canadá, y su escuela, que ya en ${ }^{(5)}$ dice que el problema se puede resolver por programación lineal o no lineal. En la ${ }^{(7)}$, que encontramos en ${ }^{(2)}$, indica que debe haber montado algún sistema de programación aplicable en ordenador.

Sin embargo, no tenemos disponible la ${ }^{(7)}$, y la ${ }^{(6)}$ todavía no ha sido enviada por la A.I.P.C. Por tanto, no sabemos cuánto de original puede tener lo que aquí se expone, $\mathrm{y}$ en todo caso nos parece útil que se imprima.

Como el preparar unos programas extensos en ordenador requiere muchos medios, hemos introducido variables auxiliares que hacen el problema calculable por programas Simplex, disponibles en todo ordenador científico moderno. En particular, la IBM tiene unos programas muy eficientes, habiendo sido pasados en España problemas de $\mathbf{1 . 0 0 0}$ variables con mil ecuaciones, según ${ }^{(9)}$. Quizás Cohn emplee métodos mucho más completos, pero más difíciles de poner en práctica.

\section{Uso del cúleulo polústico}

Si bien el cálculo límite, o cálculo en rotura, que consiste en mayorar cargas, minorar resistencias, hacer un reparto elástico de esfuerzos y comprobar secciones en rotura (o, en fin, de deformaciones reversibles para el acero), garantiza que una estructura no se cae, habida cuenta del pandeo, el cálculo plástico elemental permite decir que una es- 
tructura se cae bajo ciertas cargas, debiendo tomarse precauciones para cargas inferiores.

Según el profesor J. Martínez Calzón, que prepara una publicación sobre cálculo anelástico, materia en la que es experto, en el Instituto Torroja, las estructuras mixtas de hormigón y perfiles de acero se prestan a las hipótesis plásticas. En las metálicas siempre habrá problemas de pandeo (ver E. M. 62), salvo para estructuras bajas o estructuras intraslacionales por cruces de San Andrés, en que las vigas se pueden calcular plásticamente, no así las columnas ${ }^{(10)}$. Se usan reglamentos plásticos en los EE. UU. ${ }^{(11)}$. En hormigón las hipótesis plásticas son menos reales, aunque la ${ }^{(2)}$ estudia su aplicación, así como la ${ }^{(3)}$.

Ferry Borges y su equipo han resuelto el problema del cálculo en hormigón para leyes tensiones-deformaciones complicadas, con un programa para cualquier estructura lineal dentro de amplios límites (Laboratorio Nacional de Engenharia Civil, Lisboa).

Aun en los casos en que representa la realidad, el cálculo plástico no garantiza una seguridad contra la ruina en caso de cargas excepcionales o de sus combinaciones, pues las cargas usuales pueden producir pequeñas plastificaciones cuya repetición docenas de veces provoca la ruina de la estructura (es el «shake down»...; no se trata de la fatiga, en la que miles o millones de cargas repetidas rompen el material por debajo del límite elástico). Conviene asegurarse de que las condiciones de servicio no presentan estos problemas.

\section{Estructurus a lus que se puede uplicar el metodo pidstico}

Son las planas de barras que entran en ruina por formación de un número de rótulas suficiente para transformarlas en mecanismos totales o parciales - la rotura se produce, pues, por flexión-y que están sometidas a cargas puntuales.

Por tanto, tendrán cargas laterales importantes para que los pilares se rompan a flexión. Se pueden también estimar las cargas de los pilares, y calcular los momentos plásticos de éstos en función de estas cargas axiles, menores que los momentos plásticos a flexión pura. Al final del cálculo plástico se pueden calcular los esfuerzos axiles en rotura a partir de las rótulas que transforman la estructura en mecanismo. Con estos esfuerzos axiles se pueden ver cuáles son los momentos de rotura reales, incluso teniendo en cuenta el pandeo de las piezas, y volver a hacer un cálculo plástico. Por aproximaciones sucesivas se puede llegar a una representación real de la resistencia de la pieza (pero existen sorpresas de diversos tipos).

Se prescinde de roturas fuera del plano de la pieza. Estas roturas pueden suponer torsiones...

Las rótulas pueden producirse en los puntos de aplicación de las fuerzas puntuales que representan las cargas, y si son continuas las cargas se pueden representar por varias puntuales, siendo las dos hipótesis sólo aproximadas. Las cargas móviles pueden representarse por varias cargas puntuales en las posiciones críticas de las cargas. Pueden producirse también en los quiebros de las estructuras de las piezas rectas, y en los nudos empotrados, en número igual al de barras empotradas (menos uno, caso de haber una barra con mayor momento de rotura en ambos sentidos). Cada rótula tendrá un índice $i$. Puede, además, haber rótulas en las articulaciones (sin índice). 
Si las rótulas fuesen articulaciones y las barras rígidas, el sistema tendría $n$ grados de libertad, que es el número mínimo de fijaciones que inmovilizan la estructura. Su posición en un movimiento pequeño puede ponerse en función de $n$ parámetros $\alpha_{j}$, que pueden representar desplazamientos de un piso en estructuras traslacionales, giros de pilares, descensos por colapso local del punto de aplicación de una carga... En todo

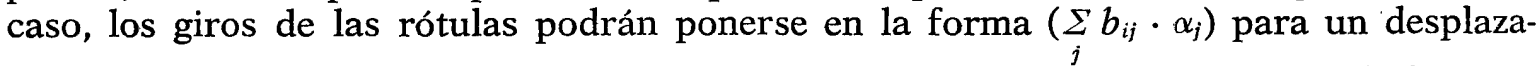
miento elemental, en función lineal de las $\alpha_{i}$. Estas $b_{i j}$, derivadas del giro de la rótula $i$ respecto al parámetro $j$, pueden obtenerse por centros instantáneos de rotación, o por otros procedimientos similares muy en favor en las Mecánicas estudiadas en Ingeniería en los últimos 50 años (Timoshenko); también puede obtenerse por equilibrios de fuerzas (método de Baker), poniendo un momento unidad en la rótula $i$, y suponiendo que en los puntos de fijación de los desplazamientos o giros $\alpha_{i}$ hay un apoyo correspondiente susceptible de dar la reacción conjugada a $\alpha_{j}$; resulta que la estructura virtual así obtenida sería isostática y de reacciones $b_{i j}$ (se demuestra por trabajos virtuales). Ver ejemplo 1 de pórtico con 4 grados de libertad. En todo caso es necesario imponer a priori un signo positivo para los $\alpha_{j}$ y para los giros $i$.

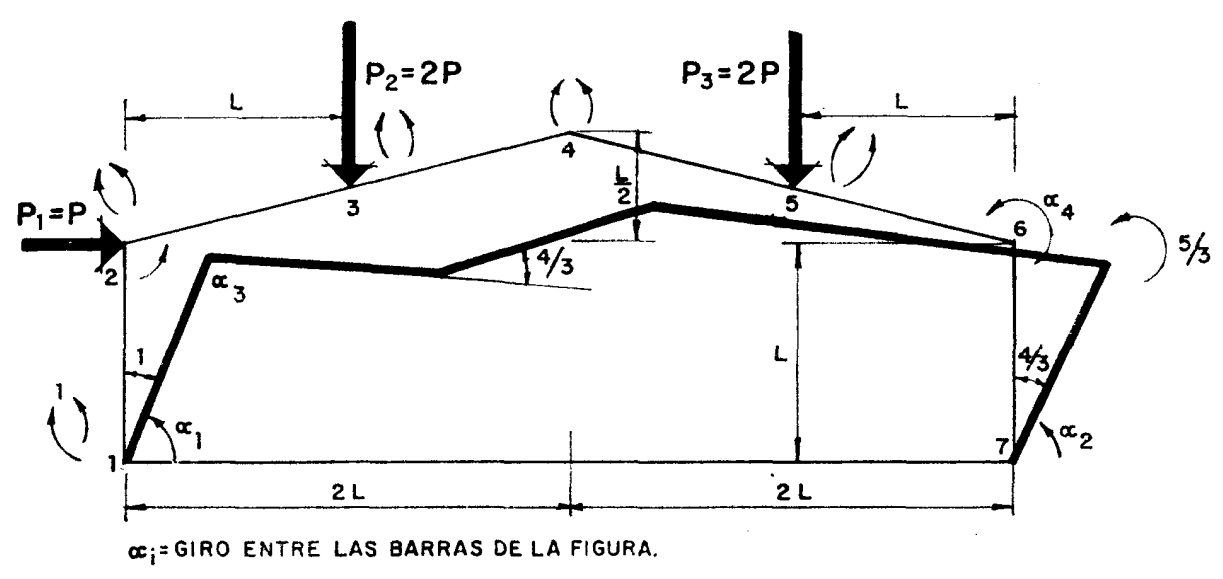

EJEMPLo 1. Pórtico plano (idéntico al de ref. 13).

El momento plástico resistente para los giros $i$ será de $M_{i}$ para giro positivo, de $N_{i}$ para giro negativo (por ejemplo, en vigas mixtas son distintos). Estos momentos pueden ser datos; algunos de ellos pueden ser incógnitas si lo que queremos es optimizar la estructura.

Todo lo anterior puede trasladarse a celosías, incluso espaciales, considerando barras $i$ plastificadas en vez de rótulas, $n$ grados de libertad, y tensiones plásticas en las barras de $M_{i}$ a tracción y $N_{i}$ a compresión (también pueden ser incógnitas). Por ejemplo, $M n_{i}=M_{i} \cdot \omega$, siendo $\omega$ el coeficiente de pandeo a compresión de la pieza.

Para celosías, ejemplo 2.

Caso particular son los entramados con cruces de San Andrés en los que los nudos se consideran rígidos, con comprobación posterior de axiles. El pandeo global se podría estudiar por programación no lineal, de la que prescindimos aquí. 
Según Lorin ${ }^{(12)}$, un dimensionamiento óptimo por métodos plásticos aprovecha mejor el material que uno elástico si el número de secciones variables es reducido (por no poder cambiar las secciones de perfiles metálicos, por ejemplo), igual si el dimensionamiento elástico se lleva a una optimización total.

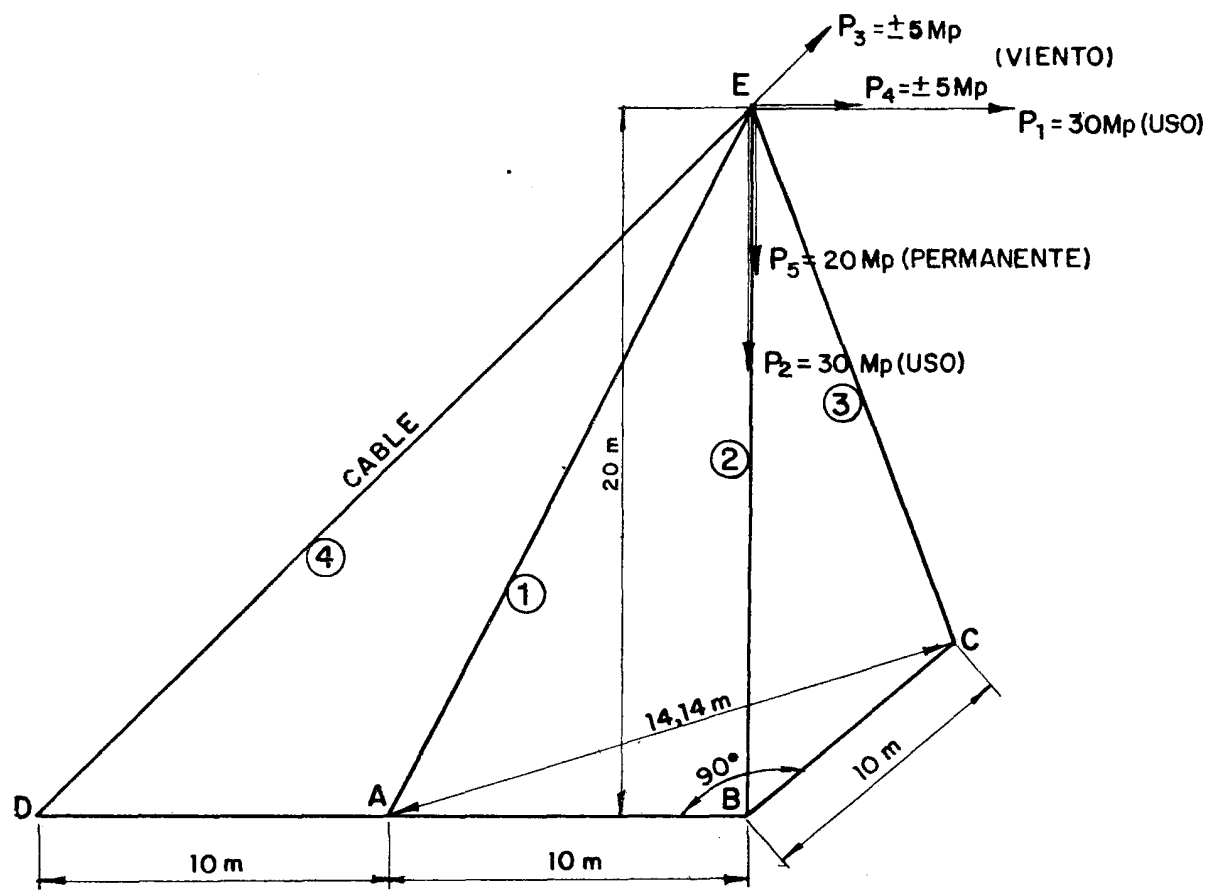

EJEMPLO 2. Soporte de andarivel (fijo).

Es necesario poner un número de fuerzas $P_{k}$ independientes de índices $k$. El trabajo de $P_{k}$ en un desplazamiento pequeño (indicado por los $\alpha_{j}$ ) puede ponerse en forma lineal:

$$
\left.\underset{j}{\left(\sum\right.} a_{j k} \cdot \alpha_{j}\right) P_{k}
$$

Los $a_{j k}$ se obtienen o por trabajos virtuales, o suponiendo las rótulas libres y calculando unas reacciones correspondientes a los desplazamientos, que serían $a_{j k}$ para $\boldsymbol{P}_{k}$ unidad.

En general una rótula se completa si $\alpha_{j}$ llega a un valor $A_{j}$, lo que permite aproximar la deformación al entrar en colapso la última rótula.

Para las rótulas ha de ser entonces mín $\left(\alpha_{j}\right)=A_{j}$. Puede ser útil considerar entonces las deformaciones elásticas y plásticas (pueden no ser despreciables las elásticas) y comprobar por un nuevo cálculo que la estructura deformada resiste, pues los esfuerzos podrían ser incrementados por la deformación (teoría de segundo orden).

El ejemplo 1 es un pórtico con dos rótulas tomado de la ${ }^{(13)}$ y el ejemplo 2 es un trípode con un viento que representa un soporte de andarivel. A las figuras corresponden cuadros de los coeficientes $b_{i j}$ y $a_{j k}$. Se ha tomado para el segundo una representación simplista de las fuerzas de viento como suma de dos fuerzas independientes: no es uniforme para cualquier rumbo su intensidad. 
En el ejemplo 1, usando centros instantáneos de rotación, los giros son $b_{i j} \cdot \alpha_{j}$ :

\begin{tabular}{r|c}
\hline Rótula $i$ & Giro respecto $\mathrm{a} \alpha_{j}\left(\right.$ son los $\left.b_{i j}\right)$ \\
\hline giro $1=$ & $\alpha_{1}$ \\
$2=$ & $\alpha_{3}$ \\
$3=$ & $-4 \alpha_{1}+2 \alpha_{2}-2 \alpha_{3}$ \\
\hline $4=$ & $5 \alpha_{1}-5 \alpha_{2}+\alpha_{3}-\alpha_{4}$ \\
$5=$ & $-2 \alpha_{1}+4 \alpha_{2} \quad+2 \alpha_{4}$ \\
$6=$ & \\
\hline $7=$ & $-\alpha_{2}$ \\
\hline
\end{tabular}

Los trabajos de las fuerzas son $\left(P_{k}\right)$ :

\begin{tabular}{|c|c|c|c|}
\hline Tipo & $k$ & \multicolumn{2}{|c|}{ Respecto al giro $\alpha_{j}$ (son los $a_{i k}$ ) } \\
\hline Viento & 1 & $-L \cdot \alpha_{1}$ & \\
\hline Uso & 2 & $-L \cdot \alpha_{1}$ & \\
\hline Uso & 3 & $+L \cdot \alpha_{2}$ & $L \cdot \alpha_{4}$ \\
\hline Permanente & 4 & Se desp & \\
\hline Permanente & 5 & Se desp & \\
\hline
\end{tabular}

El ejemplo 2 es espacial y representa un soporte de andarivel fijo:

Las tracciones son positivas, las compresiones negativas.

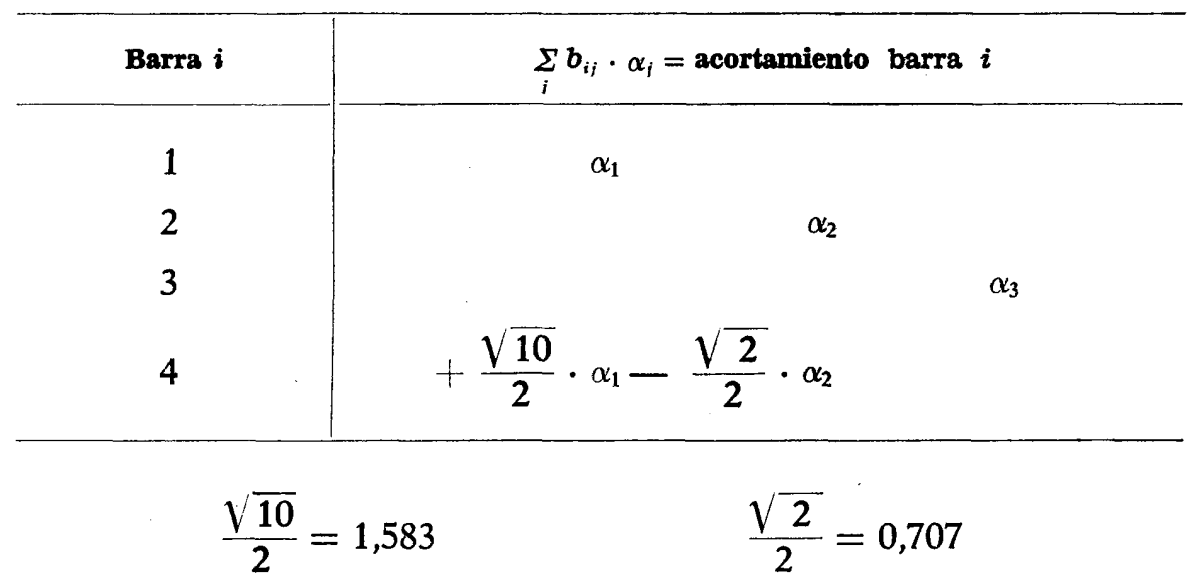




\begin{tabular}{|c|c|c|}
\hline Fuerza & $k$ & $\sum_{j} a_{i k} \cdot \alpha_{i}=$ desplazamiento fuerza $k$ \\
\hline Cable & 1 & $\sqrt{5} \cdot \alpha_{1}-2 \alpha_{2}$ \\
\hline Cable & 2 & $\alpha_{2}$ \\
\hline Viento & 3 & $+2 \alpha_{2}-\sqrt{5} \cdot \alpha_{3}$ \\
\hline Viento & 4 & $\sqrt{5} \cdot \alpha_{1}-2 \alpha_{2}$ \\
\hline Peso propio & 5 & $-\alpha_{2}$ \\
\hline
\end{tabular}

Las cargas no permanentes (uso + viento en el ejemplo) pueden ser mayoradas por coeficientes de seguridad $C_{k}$ de modo sencillo (tomamos 1,5 de coeficiente en el ejemplo), o más complicados, como ya los propuso la ACI (American Concrete Institute), debiendo resistir, por ejemplo, la estructura a la carga de viento $+1,5$ la de uso, e independientemente a la de viento por $1,5+$ la de uso por 1,2 . O bien, con ventaja, se comprobará con 1,5 la carga de uso; luego con 1,5 la carga de viento; luego con 1,3 la carga de uso y la de viento. Estos coeficientes de ponderación $C_{b k}$ tienen en cuenta la dificultad de que se produzcan a la vez vientos extremos y cargas de uso extremas. Sus valores no son más que a título de ejemplo.

Las cargas permanentes irán mayoradas por 1 o por 1,3, según sea más desfavorable.

Por tanto, habremos de considerar la peor combinación de $P_{k}$ independientes, verificando, con la primera mayoración:

- Para las no reversibles, $P_{k}$ positivo menor que $1,5 d_{k}=d_{k} \cdot C_{k}$, siendo $d_{k}$ su valor característico.

- Para las reversibles, $P_{k}$ entre $1,5 d^{\prime}{ }_{k}$ y $1,5 d_{k}$, siendo los dos valores característicos $\left(C_{k} \cdot d^{\prime}{ }_{k}\right.$ y $\left.C_{k} \cdot d_{k}\right)$.

- Para las permanentes, $P_{k}$ entre $d_{k}$ y $d_{k} \cdot C_{k}$. También puede suponerse que la permanente crece con los momentos resistentes, tomando su valor característico forma lineal $d_{k}+\sum_{i} f_{i k} \cdot M_{i}+\sum_{i} f \cdot n_{i k} \cdot N_{i}$ que reemplazaría a la usual $d_{k}$ fija. Las $f$ estarían tomadas de modo a reflejar el incremento del peso propio con la resistencia.

Con la segunda mayoración habría que hacer más comprobaciones.

Resolvemos a continuación 5 ejemplos ilustrativos sobre cuyo modelo pueden solucionarse todos los demás casos.

\section{Bomprobución elementali estructuma del ejemplo 1 con car gyas creciemalo proporcionalumente}

Da el mismo resultado que la ${ }^{(13)}$, sin tanteos.

Se suponen cargas $P_{1}=P, P_{2}=2 P, P_{3}=2 P$. Se consideran todas las rótulas con momentos $M$ iguales en ambos sentidos. 
El problema lineal a resolver es, en fuerzas:

$$
\begin{aligned}
& \text { Mín }[(-P) \cdot L / M] \\
& D_{i} \gtrless 1 \text { para } i=2,3,4,5,6 ; \\
& +8 D_{3}-10 D_{4}+4 D_{5}-3 P \cdot L / M=1 ; \\
& -4 D_{3}+10 D_{4}-8 D_{5}+2 P \cdot L / M=-1 \\
& -2 D_{2}+4 D_{3}-2 D_{4}-2 P \cdot L / M=0 \\
& +2 D_{4}-4 D_{5}+2 D_{6}+2 P \cdot L / M=0 .
\end{aligned}
$$

Las últimas ecuaciones expresan equilibrios de fuerzas. $D_{i}$ vale 0 si la rótula correspondiente actúa en sentido negativo, 1 si actúa en sentido positivo. El problema, pasado en el computador del Instituto Torroja, da los resultados:

\begin{tabular}{ccc} 
Variable & \multicolumn{1}{c}{ Valor } \\
\cline { 1 - 1 } 4 & & $0,79545454=D_{5}$ \\
1 & & $0,40909091=D_{2}$ \\
3 & & $0,77272727=D_{4}$ \\
2 & & $0,99999999=D_{3}$ \\
6 & $0,81818182=P \cdot L / M$ \\
TotaL $=$ & $0,81818045=-P \cdot L / M$.
\end{tabular}

Estos resultados indican la deformada (figura del ejemplo 1), puesto que conocemos las rótulas $\left(D_{i}=1\right.$, giro positivo; $D_{i}=0$, giro negativo).

Hallar los ángulos es ahora fácil.

El problema puede ser planteado en ángulos, y es el dual del anterior:

$$
\begin{aligned}
& -\left(3 \alpha_{1}-2 \alpha_{2}+2 \alpha_{3}-2 \alpha_{4}\right)=1 \\
& -2 \alpha_{3}+I_{1}>0 \\
& -2\left(-4 \alpha_{1}+2 \alpha_{2}-2 \alpha_{3}\right)+I_{2}>0 \\
& -2\left(5 \alpha_{1}-5 \alpha_{2}+\alpha_{3}-\alpha_{4}\right)+I_{3}>0 \\
& -2\left(-2 \alpha_{1}+4 \alpha_{2}+2 \alpha_{4}\right)+I_{4}>0 \\
& -2\left(-\alpha_{4}\right)+I_{5}>0 ; \\
& \text { Máx }\left(\alpha_{1}-\alpha_{2}+I_{1}+I_{2}+I_{3}+I_{4}+I_{5}\right) .
\end{aligned}
$$

Aún siendo dual un problema del otro, se introducen de distinto modo en el Simplex del Instituto Torroja (programado por F. Morán). En éste hay que hacer $\alpha_{i}=\alpha_{i}^{\prime}-\alpha^{\prime \prime}{ }_{i}$, con $\alpha_{i}^{\prime}$ y $\alpha^{\prime \prime}{ }_{i}$ positivas. Los resultados son:

\begin{tabular}{cll} 
Variable & & \multicolumn{1}{c}{ Valor } \\
\cline { 1 - 1 } 5 & & $0,00000000=\alpha_{3} ;$ \\
4 & & $0,36363636=-\alpha_{2} ;$ \\
7 & & $0,45454545=\alpha_{4} ;$ \\
2 & & $0,27272727=-\alpha_{1} ;$ \\
10 & & $0,36363637=I_{2} ;$ \\
TotaL $=$ & $0,81819080=P \cdot L / M$.
\end{tabular}


Según la teoría de la programación lineal, el primer término es la energía en un desplazamiento elemental (varían $\alpha_{j}$ ) cuando el parámetro vale $P$. El segundo término y el tercero son los trabajos de las rótulas, tomando $D_{i}$ un valor entre 0 y 1 , de modo que este trabajo sea lo menor posible (o mayor en valor absoluto, con signo negativo), pues siempre es negativo. El tercer término obliga, para ser máximo en $I_{i}$, a que $\left(1-D_{i}\right)$ sea negativo, o sea, $D_{i}<1$.

Para $P$ menor o igual que el $P$ crítico, el máximo sería 0 en $\alpha_{j}$.

Como este máximo no nos conviene, añadimos el último término y hacemos $P$ variable. $\boldsymbol{P}$ viene multiplicado por $\Sigma a_{j k} \cdot \alpha_{j}-1$. El pedir que la expresión sea mínima en $\boldsymbol{P}$ quiere decir que en el óptimo $\sum_{j k} a_{j k} \cdot \alpha_{j}=1$, o sea, que el trabajo de las fuerzas es $P$.

En el óptimo se anulan todos los factores salvo el último, luego el valor del problema es $P$. Hay un máximo nulo de la energía de deformación para un $P$ máximo, que corresponde al problema ingenieril. En él $P$ es el crítico, los $\alpha_{i}$ proporcionales a los giros, y los $D_{i}$ ceros o unos en las rótulas en sentido positivo o negativo, respectivamente, con valores intermedios si no hay rótula y su momento es $\left[M_{i} \cdot D_{i}-N_{i}\left(1-D_{i}\right)\right]$ en rotura.

Permiten los $\alpha_{j}$ poner ángulos proporcionales en la figura solución. El resultado es el mismo del Beedle. Al menos un programa IBM permite sacar resultados directos (primales) y duales a la vez (con ahorro de tiempo):

La formulación teórica de ambos problemas es:

$$
\left(\sum_{k} a_{j k}=a_{i 1}+2 a_{j 2}+2 a_{i 3}=\sum_{k} a_{j k} \cdot d_{k} \cdot C_{k}\right) .
$$

- Primal

Mín $(-P)$ :

- para todo $\Sigma, \underset{k}{\sum} a_{j k} \cdot d_{k} \cdot C_{k}-\sum_{i}\left[\left(M_{i}+N_{i}\right) b_{i j}\right] D_{i}+\sum_{i} N_{i} \cdot b_{i j}=0 ;$

- para todo $i, D_{i} \gtrless 1$.

- Dual

Máx $\left[\Sigma\left(N_{i} \cdot b_{i j} \cdot \alpha_{j}-I_{i}\right)\right]$ :

$\Sigma a_{j k} \cdot d_{k} \cdot C_{k} \cdot \alpha_{j}-1=0$ (o también $₹ 0$ );

- para todo $i, \sum_{j}^{j k}\left(M_{i} \cdot b_{i j}+N_{i} \cdot b_{i j}\right) \gtrless I_{i}$,

- Mixto

Máx en $\left(\alpha_{j}, I_{i}\right)$, Mín en $\left(P, D_{i}\right)$ de:

$$
\begin{aligned}
& \sum_{j k} a_{j k} \cdot d_{k} \cdot C_{k} \cdot P \cdot \alpha_{j}-\sum_{i j} M_{i} \cdot b_{i j} \cdot \alpha_{j} \cdot\left(D_{i}\right)+ \\
& +\sum_{i j} N_{i} \cdot b_{i j} \cdot \alpha_{i} \cdot\left(1-D_{i}\right)-\sum_{i} I_{i} \cdot\left(1-D_{i}\right)-P
\end{aligned}
$$

(el valor es $-P$ ). 


\section{Fomprobución con cargas variables}

Las cargas $P_{1}, \ldots P_{k}$ pueden variar entre ciertos extremos $C_{k} \cdot d^{\prime}{ }_{k}$ y $d_{k} \cdot C_{k}$.

En general, para un valor dado de los parámetros $P_{k}$, no existe un mecanismo que rompa la estructura si existe un sistema de momentos inferiores o iguales a los máximos en las rótulas equilibrando la estructura, lo que es posible multiplicando las cargas por $P$ hasta cierto valor de $P$ por encima del cual esto no es posible.

El conjunto de los $\left(P_{1}, \ldots, P_{k}\right)$ para los que la estructura resiste es el que verifica las:

- para todo $i, \quad D_{i} \gtrless 1$;

- para todo $j,-\sum_{i}\left(M_{i}+N_{i}\right) b_{i j} \cdot D_{i}+\sum_{j k} a_{j k} \cdot P_{k}+\sum_{i} N_{i} \cdot b_{i j}=0$.

Siendo $D_{i}=1$ si el momento es plástico positivo en $i, 0$ si es plástico negativo, entre 0 y 1 en caso contrario. Representa la segunda ecuación que existen $D_{i}$ (o sea momentos) tales que el movimiento según el grado de libertad $j$ es indiferente (condición necesaria y suficiente si se cumple para todo $j$ ). $\mathrm{O}$ sea, son las condiciones de equilibrio.

Representamos un caso de cargas $P_{k}$ por el vector $\left(P_{1}, \ldots, P_{n}\right)$ o punto en un espacio de $n$ dimensiones $\left(P_{k}\right)$.

Los puntos $\left(P_{k}\right)$ para los que el sistema tiene solución son proyección de la intersección de planos y semiespacios en el espacio de las $D_{i}, \ldots, P_{k}$. Como la intersección y proyección de conjuntos convexos es convexa, los $\left(P_{k}\right)$ que resiste la estructura forman un conjunto convexo en el plano $\left(P_{1}, P_{2}, P_{3}, \ldots, P_{n}\right)$.

Las condiciones de $P_{k}$ con la primera familia de coeficientes $\left(P_{k}\right.$ entre $C_{k} \cdot d^{\prime}{ }_{k}$ y $C_{k} \cdot d_{k}$ para sobrecargas, entre $d_{k}$ y $C_{k} \cdot d_{k}$ para permanentes) nos limitan un conjunto convexo de vértices, engendrado por todos los conjuntos $\left(P_{1}, \ldots, P_{n}\right)$ en los que $P_{i}$ tiene sus valores extremos $\left(C_{k} \cdot d^{\prime}{ }_{k}\right.$ y $C_{k} \cdot d_{k}$, o $d_{k}$ y $\left.C_{k} \cdot d_{k}\right)$.

Con los segundos sistemas de comprobación los vectores $\left(P_{1}, \ldots, P_{n}\right)$ mayorados también forman un sistema convexo, o por lo menos consideramos el sólido convexo mínimo que contiene sus posibles valores.

Para que el sólido convexo de cargas posibles esté dentro del sólido convexo de cargas admisibles, es necesario y suficiente que lo estén sus vértices. Comprobamos, pues, con éstos.

En el primer caso se obtienen dando a las componentes de $P_{k}$ sus valores extremos en todas las combinaciones posibles (para $n$ cargas es $2^{n}$ ). Así en el ejemplo 1 basta comprobar con los valores característicos de $P_{1}, P_{2}, P_{3}$ mayorados por:

$$
\begin{aligned}
& (0 ; 1 ; 1) \quad \text { (se puede omitir de entrada). } \\
& (0 ; 1 ; 1,3) \quad \text { (se omite por simetría con el siguiente). } \\
& (0 ; 1,3 ; 1) \quad \text { (poco probable que sea el pésimo). } \\
& (0 ; 1,3 ; 1,3) \\
& (1,5 ; 1 ; 1) \\
& (1,5 ; 1 ; 1,3) \\
& (1,5 ; 1,3 ; 1) \\
& (1,5 ; 1,3 ; 1,3)
\end{aligned}
$$


O sea, las comprobaciones son las seis últimas indicadas; según sean los momentos resistentes y los máximos de las cargas puede ocurrir cualquiera de ellas, por lo cual no existe una regla matemática sencilla que nos diga cuál es la peor, a menos de hacer un Simplex muy grande con todas las condiciones de comprobación y el mismo $P$. $O$ sea Mín $(-P)$ con las condiciones $m$ :

- para todo $i, D^{m}{ }_{i}<1$.

- para todo $j,-\sum_{i}\left(M_{i}+N_{i}\right) b_{i j} \cdot D^{m_{i}}+\sum_{j k} a_{j k} \cdot P_{k} \cdot P+\sum_{i} N_{i} \cdot b_{i j}=0$.

En cada $m$ damos a los $P_{k}$ unos valores extremos diferentes.

Los ángulos de giro en rotura se obtienen resolviendo para los $\boldsymbol{P}_{k}$ pésimos el problema dual, obteniéndose unos valores proporcionales a los giros de los ángulos en el mecanismo de rotura:

$$
\begin{aligned}
& \text { Máx }\left(\sum_{i j} N_{i} \cdot b_{i j} \cdot \alpha_{j}-\sum_{i} I_{i}\right) ; \\
& \operatorname{con} I_{i}>\sum_{j} b_{i j}\left(\alpha_{j}\right)\left(N_{i}+M_{i}\right) ; \\
& \sum_{j k} a_{j k} \cdot P_{k} \cdot \alpha_{j}=1 \text {, para los } P_{k} \text { que producen el } P \text { mínimo. }
\end{aligned}
$$

El problema mixto no ofrece interés especial.

El máximo $P$ por el que se pueden multiplicar las cargas sin llegar a condición de rotura, es el mínimo para cualquier sistema de cargas de las mínimas de cualquier sistema de $\alpha_{j}$ no nulos todos de:

$$
-\frac{\text { trabajo fuerzas internas }}{\text { trabajo fuerzas externas con } P=1}
$$

En particular, el denominador se hizo 1 para un único caso de cargas.

En cuanto haya muchos casos de carga, desbordaríamos cualquier memoria de acceso electrónico de un computador. Sin embargo, los hechos de que el último Simplex ${ }^{(2)}$ tiene coeficientes muy parecidos para cada $m$, que existen estrategias eficientes de programación rápidas que permiten operar metiendo columnas o filas en bandas magnéticas, leyendo columna a columna con pocas maniobras de las bandas, y el hecho de que a partir de pocos coeficientes $a_{j k}$ y $b_{i j}$ se puede hacer la matriz inicial por un programa sencillo, hace el procedimiento viable.

Para disminuir el gasto de computador se puede hacer un programa Simplex que deje de considerar las numerosas matrices rectangulares nulas en la primera iteración. Otro sistema es hacer los Simplex uno tras otro, en orden de seguridad aparente menor a mayor. En el primer Simplex hallamos un $P$ mínimo. En el siguiente hallamos un $P$ menor como solución del Simplex, que reemplaza a nuestro $P$ mínimo, o sobrepasamos ese valor en algún momento, y abandonamos el cálculo guardando el $P$ anterior, pasando al Simplex siguiente hasta encontrar el que dé $P$ menor.

No hemos hecho de momento ninguno de estos cambios de programación. Para un caso de cargas hemos usado un Simplex corriente sin más que rellenar la matriz de datos. Los tiempos de ejecución fueron de segundos. 


\section{Como se mesolvomín ung optimización de lou esstuructuma Iniperestática}

Es indispensable considerar varios casos de carga en general, por lo que es conveniente emplear el artificio de saltarse las matrices nulas, enunciado en el final del capítulo 5, para ahorrar tiempo y espacio de computador.

En general, hay que calcular para cada elemento un $N_{i}$ y un $M_{i}$ de resistencias a esfuerzos positivos y negativos. Si es resistencia de una sección metálica hacemos $M_{i}=N_{i}$. Si es una viga de celosía metálica hacemos $M_{i}=\omega \cdot N_{i}$, siendo $\omega$ el coeficiente de pandeo.

Consideramos sólo un $M_{i}$ por ser el $N_{i}$ función del $M_{i}$ pero se pueden poner dos variables. Aparecerá un $P_{k}$ de cargas permanentes que tomará el valor 0 y el valor de $d_{k} \cdot C_{k}$ mayorado según los casos de comprobación, pero se le puede reemplazar por $\left(d_{k}+\right.$ $\left.+\sum_{i} f_{i k} \cdot N_{i}\right) C_{k}$ si queremos que nos represente el aumento de peso propio con la resistencia.

Es necesario usar todos los casos de carga en los que las fuerzas toman valores extremos. Así, en el caso 2, haríamos una comprobación en el plano de los cables (en el otro sólo interviene $P_{3}$ ) con las cargas mayoradas por coeficientes de ponderación. Las más desfavorables se obtienen con:

\begin{tabular}{|c|c|c|c|c|}
\hline Caso $m$ & $\begin{array}{l}\text { Uso } \\
P_{1}\end{array}$ & $\begin{array}{l}\text { Uso } \\
P_{2}\end{array}$ & $\begin{array}{c}\text { Viento } \\
P_{4}\end{array}$ & $\begin{array}{c}\text { Permanente } \\
\boldsymbol{P}_{5}\end{array}$ \\
\hline 1 & 1,3 & 0 & 1,3 & 1 \\
\hline 2 & 1,5 & 1,5 & 0 & 1,3 \\
\hline 3 & 1,3 & 1,3 & 1,3 & 1,3 \\
\hline 4 & 0 & 1,5 & 0 & 1,3 \\
\hline 5 & 0 & 0 & $-1,5$ & 1 \\
\hline 6 & 0 & 1,3 & $-1,3$ & 1,3 \\
\hline 7 & & & & \\
\hline $\begin{array}{l}\text { Valores } \\
\text { característicos }\end{array}$ & $30 \mathrm{Mp}$ & $30 \mathrm{Mp}$ & $5 \mathrm{Mp}$ & $20 \mathrm{Mp}$ \\
\hline
\end{tabular}

Para los varios $m$ se establecen las restricciones en las que intervienen las cargas $P^{m}{ }_{k}$ del caso:

- para todo $i,-N_{i} \gtrless D^{m_{i}} \gtrless \omega_{i} \cdot N_{i}$;

- para todo $j, \sum_{i} D_{i}^{m_{i}} \cdot b_{i j}+\sum_{k} a_{j k} \cdot P_{k}^{m_{k}}=0$.

Y se minimiza un coste:

$$
\Sigma C_{i} \cdot N_{i}
$$

Los $C_{i}$ son la derivada del coste con respecto a los momentos resistentes. Se sabe (por ejemplo, por tanteos) lo que va a salir de estructural. $C_{i}$ es lo que cuesta incrementar en 1 el $N_{i}$ aproximadamente. 
Fácilmente pueden hacerse parte de los $N_{i}$ datos, parte incógnitas, sin más que suponerlos en las ecuaciones como parámetros en el primer caso.

Debe salir un coste mínimo con el dimensionamiento más estricto con los grados de libertad usados.

En nuestro ejemplo habría que introducir para cada caso $m$ de ${ }^{(3)}$ las $P_{k}$ mayoradas, los $a_{j k}, b_{i j}$ del ejemplo 2 , y unos $\omega_{i}$ (para el cable 0) y unos $C_{i}$ aproximados.

Son $[(3 \times 6)+3=21]$ variables con $(7 \times 6=63$ ecuaciones $)$, siendo ya recomendable el usar sistemas de programación que compongan la matriz del Simplex y prescindan de almacenar y operar con submatrices nulas, si es posible.

Sin embargo, se resuelve en caso muy general el problema de optimización de secciones de una estructura.

\section{Eonclusión}

Se expone un método de calcular estructuras mediante cálculo plástico clásico por programación lineal: el Simplex encuentra con prontitud la combinación más desfavorable de rótulas a partir de los coeficientes de Baker. Es interesante ver que el problema en giros es dual del problema en equilibrio de fuerzas.

Si bien se puede introducir este programa con cualquier Simplex, para los casos interesantes hay que preparar un programa para preparar los datos y otro para omitir las submatrices de 0 . Son estos casos:

- ver por cuánto podemos multiplicar todas las cargas para llegar a rotura en la peor combinación de casos de carga, ensayando varios casos extremos;

- ensayando todos los casos posibles de carga se puede hacer un dimensionamiento óptimo de la estructura, repartiendo las resistencias entre una parte de los elementos no dimensionados todavía, o la totalidad, minimizando el costo de material.

Para un caso de cargas es cuestión de minutos de computador el resolver un problema de 50 rótulas y 50 grados de libertad, recomendando el hacer un pequeño programa que forme la matriz inicial del Simplex, por comodidad.

\section{Obscrucación}

Posteriormente a la redacción del artículo, el Sr. Rasilla, del Servicio de Información Bibliográfica del Instituto Eduardo Torroja, nos envió las ${ }^{(15)}$ y ${ }^{(16)}$. En ellas Carlo Gavarini y Giulio Ceradini habían explotado ya un método bastante similar, dando el método teórico para utilizarlo en el estudio del pandeo en plasticidad y de emplearlo en teorías probabilistas de seguridad (con programación estocástica). No estudian con él optimización de estructuras y usan símbolos algo distintos. 
Tenemos también noticia de que un alumno de la Escuela de Caminos presentó independientemente, y sin saber programación lineal, algo sobre este tema.

Los artículos italianos mencionados son interesantes, ya que admiten leyes de plastificación más complicadas; pensábamos explotar esta posibilidad antes de conocerlos.

\section{Bibliografáa}

1. Dusan Krajcinovic. Limit analysis of structures, Proceedings A.S.C.E., Structural Division, Sept. 69.

2. M. Z. Cohn, E. F. P. Burnet, D. E. Grierson. Safety, Serviceability and Efficiency of Limit Design for Reinforced Concrete Beams and Frames, Mémoires de l'A.I.P.C., 1969 (29-I).

3. F. Sawko, A. M. B. Wilde. Large deflexion Effects in Elasto-Plastic Frame analysis, Mémoires de l'A.I.P.C., 1969 (29-I).

4. A. L. L. BAKER. Ultimate load design of reinforced concrete frames. A recapitulation and appraisal. March 1963. Publications I.A.B.S.E., V-23, 1963.

5. M. Z. Cohn. Limit design of reinforced concrete frames. Proceedings A.S.C.E. Journal of Structural Division, ST 10, Oct. 68.

6. M. Z. CoHn and D. E. Grierson. Optimal design of reinforced concrete beams and frames. Final publication. 8th I.A.B.C.E. Cougress, New York, Sept. 68.

7. M. Z. CoHN and D. E. GRIERson. An automatic approach to the analysis of plastic frames under fixed und variable loading. Paper presented at the A.S.C.E. National Meeting on Structural Engineering.

8. En prensa en la Revista de Obras Públicas. Un artículo sobre resolución del método de plasticidad elemental.

9. Economía Industrial, octubre 69, D. Carlos Mendoza Gimeno, sobre aplicación de la programación lineal a la planificación de la energía española.

10. J. YURA, Le Wu Lu. Proceedings A.S.C.E., ST 10, Oct. 69, Ultimate load tests on braced multistory frames.

11. Lynn S. Beedle, Le Wu Lu, Lee Chong Lim. Recent developments in plastic design practice. Proceedings A.S.C.E., ST 9, Sept. 69.

12. P. LoRIN. En la publicación definitiva del Congreso de la A.I.P.C., Nueva York, 1968, aparecerá un artículo sobre comparación de diseños óptimos plástico y elástico.

13. BEEDLE, L. Diseño plástico de marcos de acero.

14. S. Ferry Borges. Publicaciones del Laboratorio Nacional de Engenharia Civil de Lisboa.

15. Carlo Gavarini. Programación lineal aplicada a plasticidad. Atti dell Istituto di Scienza delle Construzioni della Universita di Pisa. Vol. IX, 1968.

16. Giornali del Genio Civili, Ag. 69. Dos artículos: uno de Givlio Ceradini y Carlo Gavarini, Applicazione della programmazione lineare ai problemi di adattamento plastico statico o dinamico; otro de Carlo Gavarini, Concezione probalistica del calculo a rotura. 


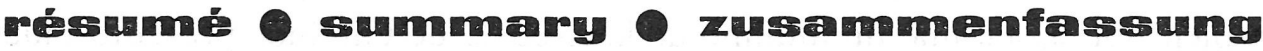

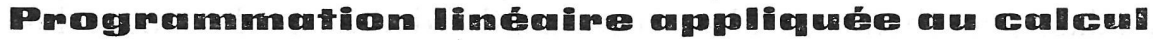 \\ polvshiqu
}

\section{J. M. Antón Corrales, ingénieur des Ponts et Chaussées}

L'auteur expose dans cet article une méthode permettant d'effectuer des calculs plastiques élémentaires des structures linéaires à charges ponctuelles, à l'aide de programmes Simplex, déjà disponibles dans tout ordinateur scientifique. Ces calculs ont pour résultat, soit un coefficient de sécurité d'une structure déjà dimensionnée, soit une optimisation de celle-ci. Le calcul est très court pour un seul cas de charges, moins pour plusieurs. La méthode admet des perfectionnements, qui peuvent rendre plus élémentaire encore son introduction dans un ordinateur.

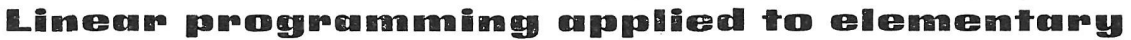

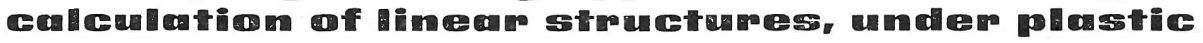 com dintions}

\section{J. M. Antón Corrales, civil engineer}

A method is described for elementary calculations of linear structures, under non elastic conditions, and subjected to point loads. The method involves the use of Simplex programs, which are already available for any scientific computer. The result gives either a factor of safety for a structure that has already been designed, or else an optimum design for given conditions. The calculation is very short for one loading system, and less so, if several loading conditions are assumed. The method can be further improved, thus rendering its use even more simple.

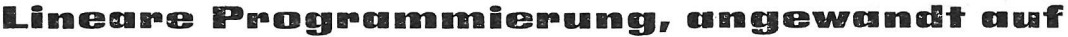

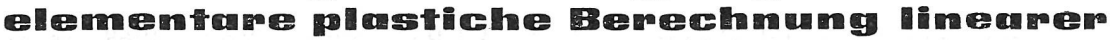

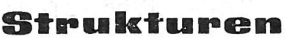

\section{J. M. Antón Corrales, Bauingenieur}

In diesem Artikel wird eine Methode beschrieben zur elementaren plastischen Berechnung linearer Strukturen mit Spitzenbelastungen mit Hilfe der SimplexProgramme, die bei jedem wissenschaftlichen Computer bereits zur Verfügung stehen; als Resultat ergibt sich entweder ein Sicherheitskoeffizient einer bereits dimensionierten Struktur, oder eine Optimierung derselben. Die Berechnung ist sehr kurz bei einem Fall von Lasten und nicht so kurz bei mehreren Fällen von Lasten. Diese Methode kann noch weiter perfektioniert werden, wodurch die Eingabe in den Computer sich noch einfacher gestaltet. 